\title{
On-road Emission Characteristics of Logistics Transportation Vehicles in Chengdu
}

\author{
Qi-Jun ZHANG ${ }^{1, a}$, Lin WU $^{1, b}$,Xiang LIU ${ }^{2, c}$, Kai-Shan ZHANG ${ }^{2, d}$, \\ Hong-Jun MAO ${ }^{1, \mathrm{e},{ }^{*}}$ \\ ${ }^{1}$ College of Environmental Science and Engineering, Nankai University, Tianjin, China \\ ${ }^{2}$ College of Architecture and Environment, Sichuan University, Chengdu, Sichuan, China

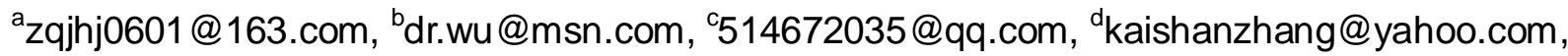 \\ ehongjun_mao@hotmail.com \\ ${ }^{*}$ Corresponding author
}

Key words: Diesel vehicles; PEMS; SEMTECH-ECOSTAR; Chengdu;

\begin{abstract}
On-road vehicle tests of three diesel trucks were conducted by a portable emission measurement system (PEMS) in Chengdu, China. SEMTECH-ECOSTAR provided by Sensors, Inc and MI2, an emissions measuring instrument powered by the Pegasor Particulate Sensor (PPS) were employed to detect gaseous and particle emissions during the tests. The impacts of speed and acceleration on emissions were analyzed. The average NOx emission factors of the heavy duty diesel truck (HDDT), medium-duty diesel truck (MDDT), light duty diesel truck (LDDT) were 7.29, 5.29 and $5.53 \mathrm{~g} / \mathrm{km}$, all of which were in excess of their emission limits. The particle emission factors were $0.60,0.30$ and $0.14 \mathrm{~g} / \mathrm{km}$, generally higher than the results for similar reported in the previous studies. Both gaseous and particle emission exhibit significant correlations with the change in vehicle speed, acceleration and power demand. The highest emission for each vehicle were generally found in high-VSP. High engine power caused by aggressive driving or heavy load is the main factor of high emissions for the vehicles in real-world situation.
\end{abstract}

\section{Introduction}

Due to their high toxicity, adverse health effects and their abundance near the roads, particularly in urban areas, diesel emissions are of high concern in recent decades. Gaseous emissions from vehicles, including NOx, THC, and $\mathrm{CO}$, have been as the primary precursors of photochemical smog. Fine particles have attracted increasing attention due to their strong impact on aerosol formation[1, 2] . PM and NOx emissions from vehicles account for about $50 \%$ of emission inventories[3]. This study focuses on the real-world operating condition and emission characteristics of diesel trucks for logistics transportation. This research was motivated by the recognition that in most Chinese cities, diesel trucks are the most widely used logistics transportation. The diesel trucks are the major contributors to the urban environmental pollution.

To better understand the emission characteristics of diesel vehicles, many measurements have been conducted in recent years. Emissions measurements for diesel vehicles have been made using chassis and engine dynamometer, remote sensing, tunnel studies and on-board measurements (PEMS) $[4,5]$. PEMS has been developed as an effective and practical method to quantify vehicle emission levels with real-world vehicle activities [6, 7]. Previous studies have conducted numerous tests to measure the emissions from different type vehicles using PEMS, which revealed that vehicle emissions are strongly related with driving cycle, fuel quality, and after-treatment equipment [8, 9]. PEMS testings have also been carried out in Chinese cities [10-12]. These studies have produced many results for in-use vehicle emission characteristics and have laid a foundation for policy-making in vehicle pollution control in China. A few works have studied impacts of vehicle speed and acceleration on vehicle emissions.

For the time being, most published studies on real-world vehicle emissions focuses on 
automobiles[10, 13], however very little work have been published on urban logistics transportation vehicles. The purpose of the current research was to survey correlation of pollutant emissions and driving conditions. Three Euro III emission level trucks were chosen for this survey, because most of Chengdu trucks are using this type engine after renewed in the year 2008. In this study, high-precision vehicle emissions testing system (PEMS) was used to get the logistics vehicles emissions data under actual operating conditions in Chengdu, calculate the emission factor, and analyze such vehicles emission characteristics.

\section{Materials and methods}

\section{Tested trucks}

The on-road emission tests were conducted in 2014 in Chengdu. Several vehicle types were selected in this study, including a heavy duty diesel truck (HDDT), medium-duty diesel truck(MDDT), light duty diesel truck(LDDT),. The specifications of the test vehicles were listed in Table 1. Each vehicle type satisfies the latest emission standards in Chengdu. The diesel fuel used in the test were obtained directly from the petrol station. The fuel quality met the requirements of the local standard equivalent to Euro 3. The sulfur content of diesel fuel should be controlled below $0.035 \%$.

Table 1 Test vehicle specifications.

\begin{tabular}{llll}
\hline Vehicle type & $\begin{array}{l}\text { heavy duty } \\
\text { diesel truck }\end{array}$ & $\begin{array}{l}\text { medium-duty } \\
\text { diesel truck }\end{array}$ & $\begin{array}{l}\text { light duty } \\
\text { diesel truck }\end{array}$ \\
\hline Fuel type & Diesel & Diesel & Diesel \\
GVWR (kg) & 16000 & 8100 & 4320 \\
Engine type & Diesel engine & Diesel engine & Diesel engine \\
Displacement (L) & 3.9 & 2.77 & 2.77 \\
Emission standard & Euro 3 & Euro 3 & Euro 3 \\
Model year & 2011 & 2010 & 2010 \\
Mileage traveled $(\mathrm{km})$ & 60907 & 133734 & 102096 \\
\hline
\end{tabular}

\section{On-road test routes}

Several papers have discussed the effects of driving condition on gaseous emissions and particulate emissions from diesel vehicles. The test route for on-board measurement was designed to simulate real traffic conditions in suburb of Chengdu (Figure 1). The total distance of the test route was approximately $17 \mathrm{~km}$. Each tested vehicle was driven thrice per day. Logistics park is located in the southwest of test section.

\section{Measurement system}

The gaseous emission analyzer was SEMTECH-ECOSTAR that collects instantaneous and accumulated data under real-driving conditions. $\mathrm{CO}$ and $\mathrm{CO} 2$ were measured by Non-dispersive infrared method(NDIR). NOx measurement, including NO and NO2, was based on Non-dispersive ultraviolet theory(NDUV). The hydrocarbon components(THC) in the exhaust stream was detected by Flame Ionization Detector subsystem(FID). Precisions of CO, CO2, THC, NOx test were 10 ppm, $0.01 \%, 1 \mathrm{ppm} \mathrm{C}$ and $1 \mathrm{ppm}$, respectively. Resolution time of the emission analyzer and $\mathrm{PM}_{2.5}$ monitor was one second level. Speed, location and altitude information of the test vehicle was collected by GPS module integrated in SEMTECH-ECOSTAR.

The $\mathrm{PM}_{2.5}$ monitor was Mi2 that is powered by the Pegasor Particulate Sensor(PPS). The PPS records PM via diffusion charging of the sampled aerosol. The operating principle is escaping charge that the current induced as the charged aerosol exits, designed as a Faraday cage[14, 15]. This current is related to active surface area. Some auxiliary information, as mean size, is needed to 
calculate particle number and PM mass. The sample aerosol is drawn from the vehicle exhaust pipe by a pump at $4 \mathrm{~L} / \mathrm{min}$ inlet flow, as adjusted by the pressure of air supplied. The sample inlet path must be heated to $190{ }^{\circ} \mathrm{C}$, and the sensor outlet is connected back to the exhaust pipe.

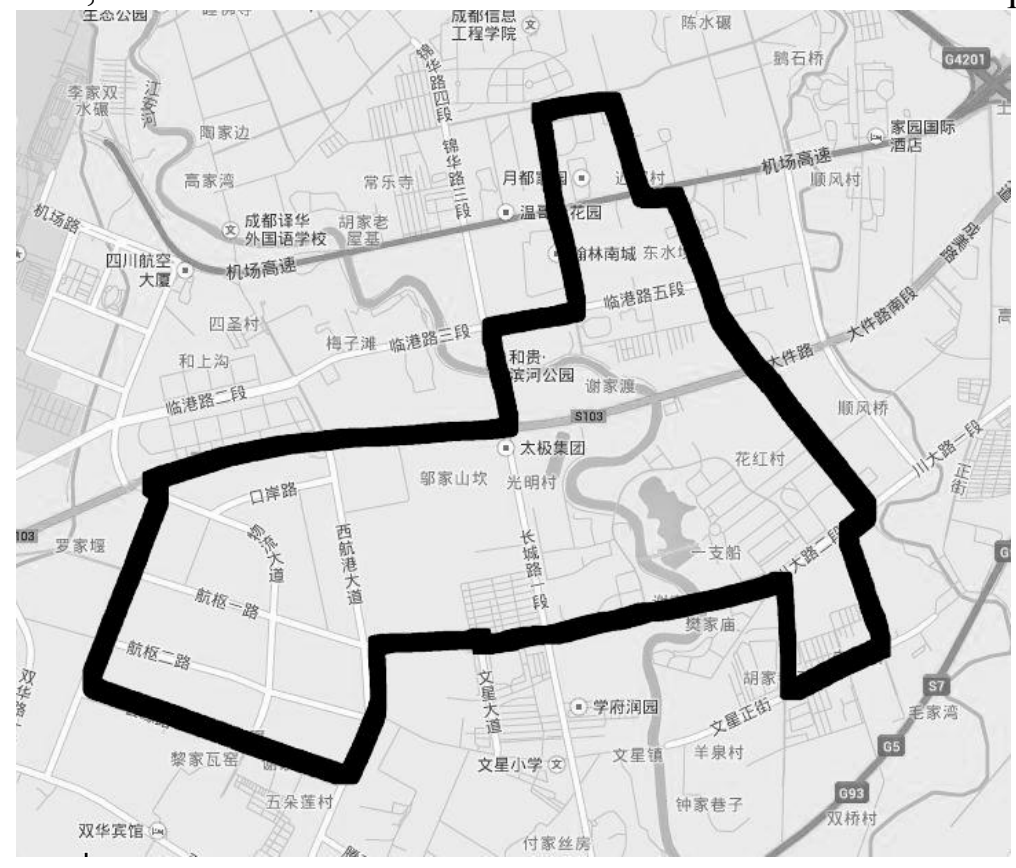

Figure 1 The test route for on-board measurement

\section{Quality assurance and statistical analysis}

Gas analyzer (SEMTECH-ECOSTAR), $\mathrm{PM}_{2.5}$ monitor (MI2), and other instruments were maintained in good technical status. The gas analyzers and $\mathrm{PM}_{2.5}$ monitor were regularly calibrated and checked for precision over the range of measurements.

At the beginning of each test, a series of preparing procedures were performed, including warming-up the vehicle, SEMTECH-ECOSTAR, MI2, calibration and leak check for the measurement devices. Calibration of the on-vehicle emission gas analyzer and $\mathrm{PM}_{2.5}$ monitor employed three kinds of samples, they were $\mathrm{N}_{2} ; \mathrm{NO}_{2}$ mixed with $\mathrm{He}$; a blend of $\mathrm{THC}, \mathrm{CO}, \mathrm{CO}_{2}$, $\mathrm{NO}$ and $\mathrm{N}_{2}$. After warming-up and calibration, the test equipment was adjusted to measure the exhaust stream of the test vehicle.

Vehicle specific power (VSP) is consistently identified as a proxy variable for engine load that was highly correlated with emissions. VSP is defined as the engine power output per vehicle unit mass and is expressed as a function of vehicle speed, road grade and acceleration. VSP for trucks was estimated using typical coefficient values and described in Zhai's work[16].

The regression coefficient of calibration standards of different pollutants was $>0.999$. Statistical data analysis (e.g., standard deviation, mean, maximum and minimum concentrations) was performed using Origin 8.5.

\section{Results and discussion}

\section{On-road driving-based emissions}

For the three type diesel vehicles selected for on-road testing, 7200 second-by-second data points were obtained, consisting of engine load, vehicle speed, acceleration, exhaust gas flow, exhaust temperature, exhaust density, $\mathrm{CO}, \mathrm{THC}, \mathrm{NO}, \mathrm{NO}_{2}, \mathrm{NOx}$, and $\mathrm{PM}_{2.5}$ concentrations(both mass and number concentrations). Figure 2 provides examples of raw emission data profiles from a single truck (No.2, medium-duty diesel truck), illustrating the temporal variations of speed, acceleration, $\mathrm{CO}, \mathrm{THC}, \mathrm{NOx}$ and $\mathrm{PM}_{2.5}$ mass concentrations under real driving conditions. Vehicle emissions are 
strongly related to engine load, vehicle speed, and acceleration; however, even under the same engine load, vehicle speed and acceleration conditions, the four pollutant emissions can be quite different. The relationship between the gaseous and particulate emission and average speed on road is similar to that found in previous PEMS study in Shanghai and Beijing.

As can be seen in Table 2, CO and THC are not significant emission species in most diesel vehicles, which are usually low and most of diesel engine manufacturers can easily meet $\mathrm{CO}$ and THC regulations.
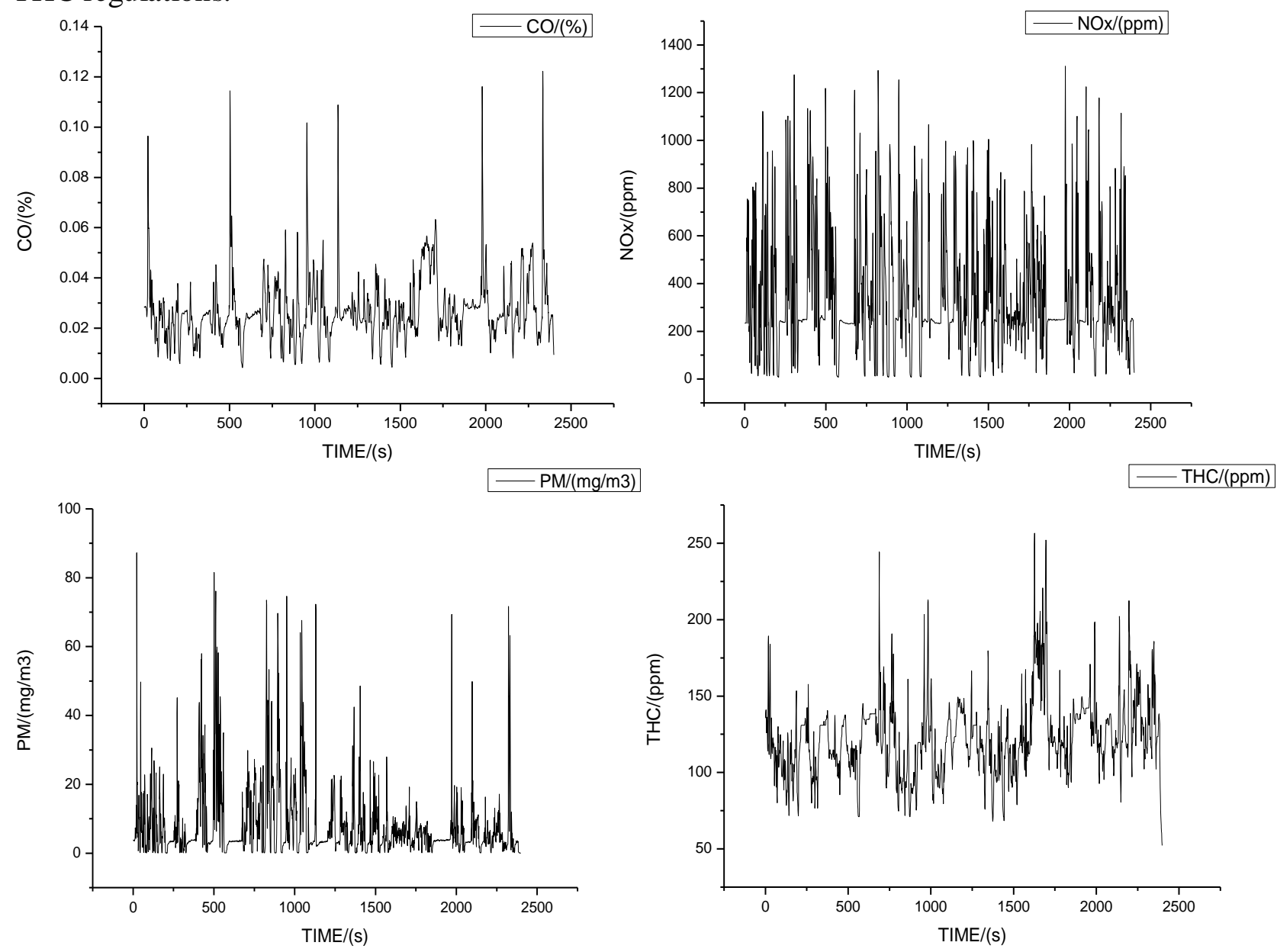

Figure 2 Typical raw simultaneous measurement data of CO concentration, THC concentration, NOx concentration and $\mathrm{PM}_{2.5}$ concentration.

Table 2 On-road driving-based emission factors of the tested vehicles

\begin{tabular}{ccccc}
\hline \multirow{2}{*}{ Vehicle type } & \multicolumn{4}{c}{ Emission factor } \\
\cline { 2 - 5 } & $\mathrm{CO}(\mathrm{g} / \mathrm{km})$ & $\mathrm{NOx}(\mathrm{g} / \mathrm{km})$ & $\mathrm{THC}(\mathrm{g} / \mathrm{km})$ & $\mathrm{PM}(\mathrm{g} / \mathrm{km})$ \\
\hline HDDT & 5.69 & 7.29 & 0.20 & 0.60 \\
MDDT & 2.10 & 5.29 & 0.24 & 0.30 \\
LDDT & 1.73 & 5.53 & 0.32 & 0.14 \\
\hline
\end{tabular}

\section{Effect of acceleration on emissions}

Emission rate of pollutants is pollutant mass per unit of time, the unit is g/s. Its numerical size and vehicle emission levels are associated with operating conditions. The relationship between acceleration (ACC) and CO, THC, NOx and $\mathrm{PM}_{2.5}$ emissions rate from a testing diesel vehicles ((No.2, medium-duty diesel truck) are shown in Figure 3.

Condition of vehicle emissions is associated with the driving condition of the vehicle. Under the same acceleration, vehicle speed is not necessarily the same, which is corresponding to the different 
pollutant emission rates. Figure 3 shows that the testing vehicle emissions rate is mainly concentrated in the acceleration range -1 to $1 \mathrm{~m} / \mathrm{s} 2$. The variation range of CO, NOx, THC and $\mathrm{PM}_{2.5}$ emission concentration is mainly concentrated in the 0 to $0.12 \%, 0$ to $1310.9 \mathrm{ppm}, 0$ to 256.6 ppm, 0 to $87.28 \mathrm{mg} / \mathrm{m} 3$, respectively.

In deceleration conditions, emission rates of CO, THC, NOx and $\mathrm{PM}_{2.5}$ pollutants are in low level. Pollutant emission rate in accelerated conditions is significantly greater than the emission rate of deceleration conditions. Fuel combustion is not complete with higher acceleration situation, resulting in the greater emission rate of pollutants. Therefore, the occurrence frequency of sudden acceleration can be reduced in the process of driving, which can effectively cut down diesel vehicle exhaust emissions.
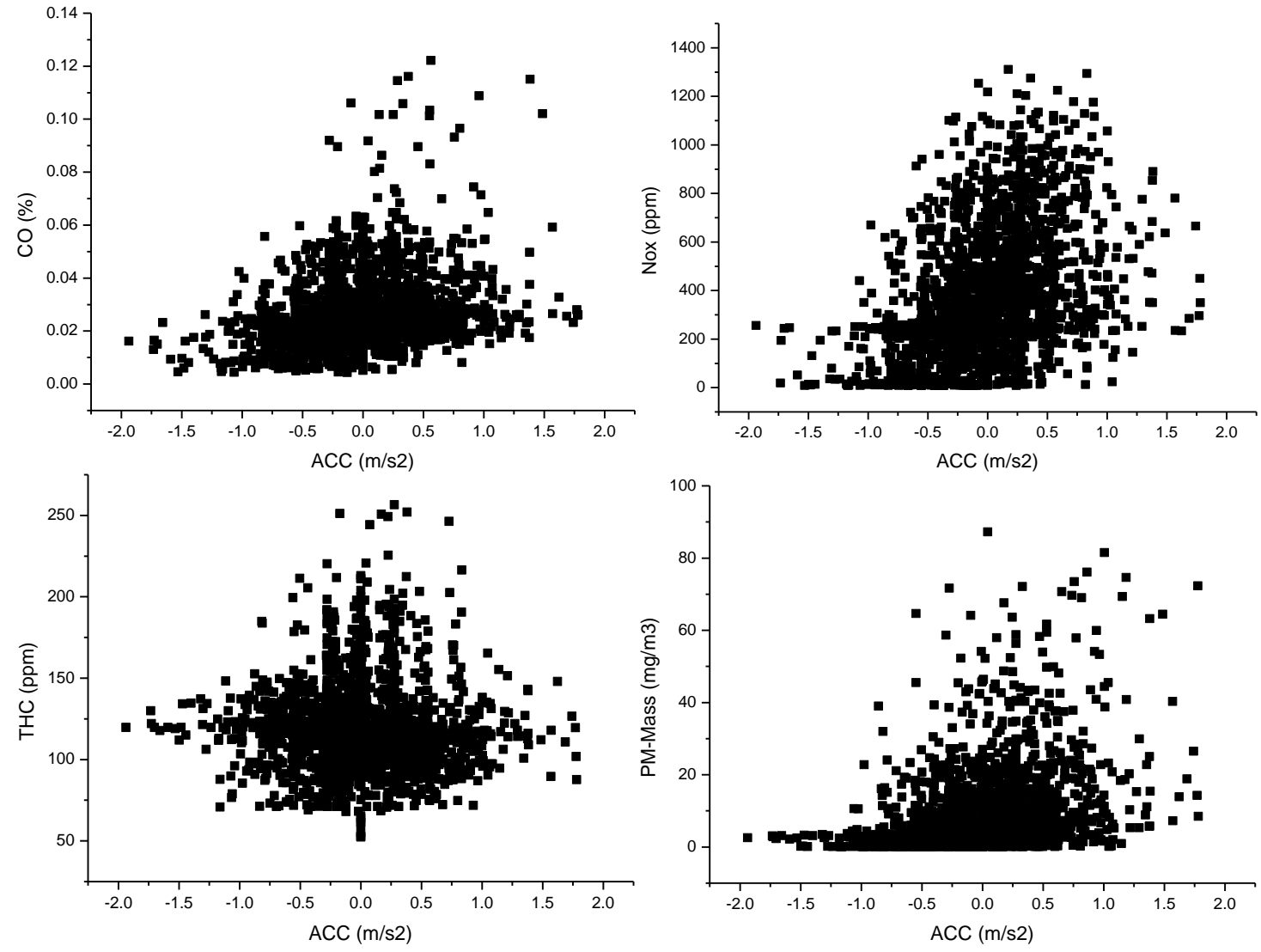

Figure 3 Relationship among acceleration, deceleration, and emission rates.

\section{Effect of driving condition on emissions}

The correlation of pollutants and the driver driving condition can not be verified by acceleration, so the VSP is particularly important. Vehicle specific power (VSP) is defined as the power demand per unit vehicle mass. In this study, we use VSP to indicate driving conditions to observe their correlation with gaseous and particle emissions.

Figure 4 shows the gaseous emission concentration of the from a testing diesel vehicles (No.2, medium-duty diesel truck) under different driving conditions. The results show that the gaseous emission rates of each vehicle increase with increasing VSP value, with the trends being similar to those in the MOVES model and previous studies. However, the highest emission rates occur in different VSP value for vehicles. The NOx and THC emission rates of the vehicle are highest in the high-VSP. As for CO, it had a positive correlation with VSP as well, but when VSP was kept at high level, CO emission dropped. Starting and accelerating under heavy load conditions is the main cause of its high emission. For the diesel vehicles, the gaseous emission rates are mainly affected by engine power instead of speed. Figure 4 also shows the particle emission rates of the tested vehicles in various VSP. The maximum particle emission rates are located in different VSP. For the test 
vehicle, its particle emission rate peaks in the highest VSP. When VSP $<0$, PM changes with VSP more smooth and when the VSP > 0, PM emissions and VSP is a linear growth. linear growth. In general, high engine power caused by aggressive driving or heavy load is the main factor of high emissions for the vehicles in real-world situation.
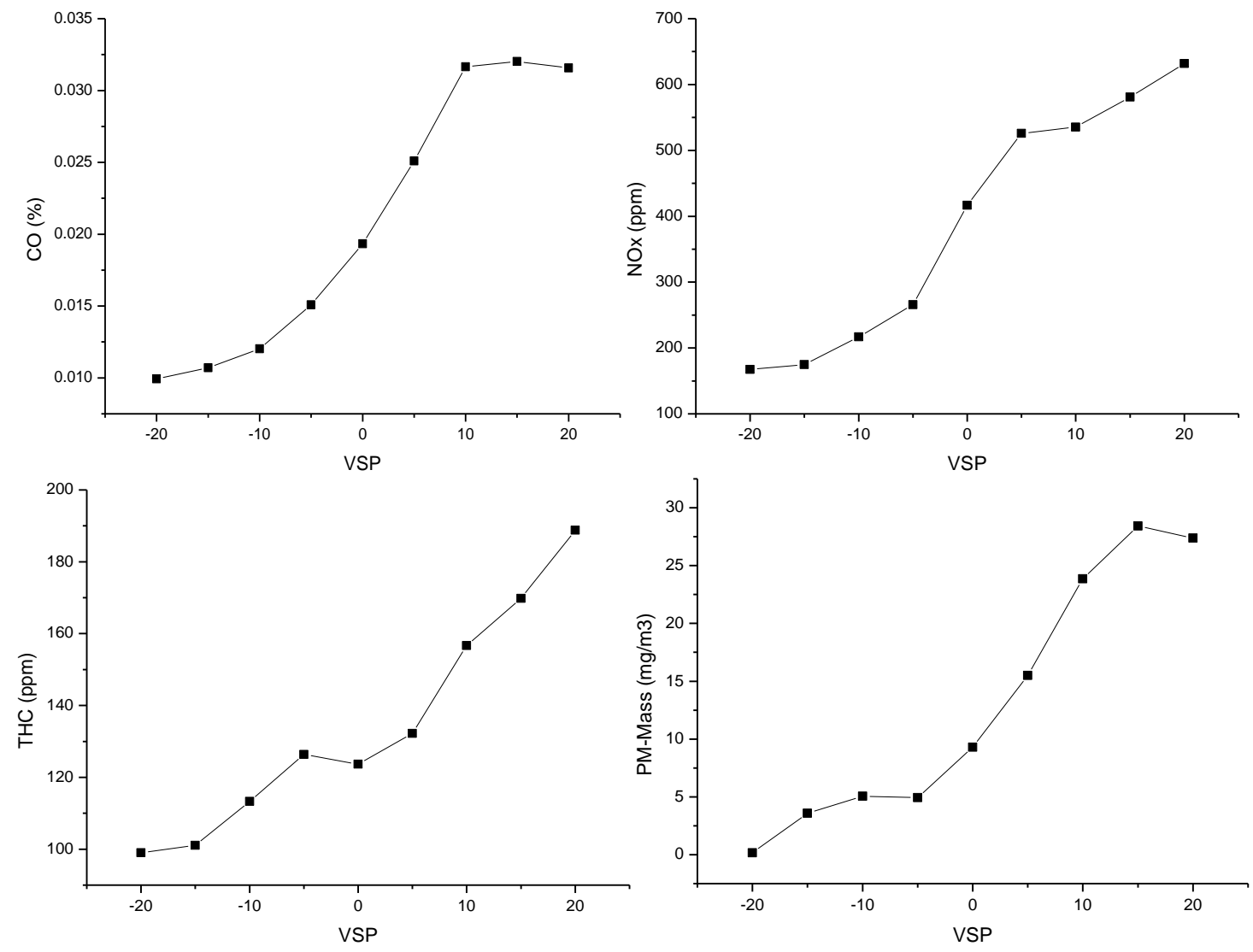

Figure 4 Emission concentration vs vehicle-specific power.

\section{Conclusions}

Diesel vehicles were tested using a PEMS system to study on the gaseous and particulate emission in Chengdu, China. The results indicate that NOx emission control still remains a significant issue for these vehicles in China. The particle emissions of the tested vehicles are generally higher than those of similar vehicle types in previous studies. The particle emission factors of the three tested vehicles are on the same order of magnitude. More attention needs to be paid to the contribution of nanoparticles from diesel exhaust to the formation of organic aerosols in the atmosphere.

Both the gaseous pollutants and particles of the tested vehicles are strongly related with their real-world driving conditions. The maximum emissions of the diesel vehicles appear in the high-VSP. The emissions of the diesel vehicles are mainly affected by engine power regardless of vehicle speed. High engine power caused by aggressive driving or heavy loads is the main contributor to high emissions for these vehicles in real-world situations.

The results of this research were also compared with the similar researches. It is noteworthy that much larger amount of CO, NOx, THC and PM pollutions were exhausted by a vehicle on different operating. It should be noted that only one vehicle of each type was tested, which is not sufficient to reflect the general emission characters of the vehicles. More studies must be conducted to clarify the each vehicle type's emission characteristics in real-world conditions. 


\section{Acknowledgement}

This research was financially supported by the National High-tech Research and Development Program (863 Program 2012AA063303) and China Europe energy conservation and emission reduction of small and medium-sized enterprises scientific research cooperation projects (SQ2013ZOA100003).

\section{References}

[1] FINLAYSON-PITTS B J, PITTS J N. Tropospheric air pollution: ozone, airborne toxics, polycyclic aromatic hydrocarbons, and particles [J]. Science, (1997), 276: 1045-1051.

[2] ZHANG R. Getting to the critical nucleus of aerosol formation [J]. Science, (2010), 328: 1366-1367.

[3] NEL A. Air pollution-related illness: effects of particles [J]. Science, (2005), 308: 804-806.

[4] YANOWITZ J, MCCORMICK R L, GRABOSKI M S. In-use emissions from heavy-duty diesel vehicles [J]. Environmental science \& technology, (2000), 34: 729-740.

[5] KADO N Y, OKAMOTO R A, KUZMICKY P A, et al. Emissions of toxic pollutants from compressed natural gas and low sulfur diesel-fueled heavy-duty transit buses tested over multiple driving cycles [J]. Environmental science \& technology, (2005), 39: 7638-7649.

[6] DURBIN T D, JOHNSON K, COCKER D R, et al. Evaluation and comparison of portable emissions measurement systems and federal reference methods for emissions from a back-up generator and a diesel truck operated on a chassis dynamometer [J]. Environmental science \& technology, (2007), 41: 6199-6204.

[7] ZHANG K, FREY C. Evaluation of response time of a portable system for in-use vehicle tailpipe emissions measurement [J]. Environmental science \& technology, (2007), 42: 221-227.

[8] DURBIN T D, JOHNSON K, MILLER J W, et al. Emissions from heavy-duty vehicles under actual on-road driving conditions [J]. Atmospheric environment, (2008), 42: 4812-4821.

[9] JOHNSON K C, DURBIN T D, JUNG H, et al. Quantifying in-use PM measurements for heavy duty diesel vehicles [J]. Environmental science \& technology, (2011), 45: 6073-6079.

[10] CHEN C, HUANG C, JING Q, et al. On-road emission characteristics of heavy-duty diesel vehicles in Shanghai [J]. Atmospheric environment, (2007), 41: 5334-5344.

[11]LIU Z, GE Y, JOHNSON K C, et al. Real-world operation conditions and on-road emissions of Beijing diesel buses measured by using portable emission measurement system and electric low-pressure impactor [J]. Science of the Total Environment, (2011), 409: 1476-1480.

[12] WU Y, ZHANG S, LI M, et al. The challenge to NO x emission control for heavy-duty diesel vehicles in China [J]. Atmospheric Chemistry and Physics, (2012), 12: 9365-9379.

[13] SAWANT A A, SHAH S D, ZHU X, et al. Real-world emissions of carbonyl compounds from in-use heavy-duty diesel trucks and diesel Back-Up Generators (BUGS) [J]. Atmospheric environment, (2007), 41: 4535-4547.

[14] MAMAKOS A, BONNEL P, PERUJO A, et al. Assessment of portable emission measurement systems (PEMS) for heavy-duty diesel engines with respect to particulate matter [J]. Journal of Aerosol Science, (2013), 57: 54-70.

[15] MARICQ M M. Monitoring Motor Vehicle PM Emissions: An Evaluation of Three Portable Low-Cost Aerosol Instruments [J]. Aerosol Science and Technology, (2013), 47: 564-573. 
[16]ZHAI H, FREY H C, ROUPHAIL N M. A vehicle-specific power approach to speed-and facility-specific emissions estimates for diesel transit buses [J]. Environmental science \& technology, (2008), 42: 7985-7991. 